\title{
Linx
}

Revue des linguistes de l'université Paris X Nanterre

$82 \mid 2021$

Entre vieillissement et innovation : le changement linguistique

\section{Le réinvestissement d'expressions datées dans le discours médiatique : le cas du syntagme Bête du Gévaudan}

New uses of old expressions in contemporary media discourses: the case of the French syntagm Bête du Gévaudan

\section{Marie Chandelier}

\section{OpenEdition}

\section{Journals}

Édition électronique

URL : https://journals.openedition.org/linx/8075

DOI : 10.4000/linx.8075

ISSN : 2118-9692

\section{Éditeur}

Presses universitaires de Paris Nanterre

Référence électronique

Marie Chandelier, «Le réinvestissement d'expressions datées dans le discours médiatique : le cas du syntagme Bête du Gévaudan », Linx [En ligne], 82 | 2021, mis en ligne le 15 juillet 2021, consulté le 21 juillet 2021. URL : http://journals.openedition.org/linx/8075; DOI : https://doi.org/10.4000/linx.8075

Ce document a été généré automatiquement le 21 juillet 2021.

Département de Sciences du langage, Université Paris Ouest 


\title{
Le réinvestissement d'expressions datées dans le discours médiatique : le cas du syntagme Bête du Gévaudan
}

New uses of old expressions in contemporary media discourses: the case of the French syntagm Bête du Gévaudan

\author{
Marie Chandelier
}

\section{Introduction}

En relayant des événements, la presse écrite consacre à certains faits d'actualité une forte production de discours et constitue ainsi des moments discursifs (Moirand, 2007a). Ces moments discursifs peuvent donner lieu à l'émergence d'expressions référant aux événements, qui font l'objet d'un usage soutenu, diversifié, avant de se stabiliser en discours ou de disparaître. De tels moments discursifs rendent ainsi possible l'étude des phénomènes d'émergence et de disparition des usages. C'est particulièrement le cas pour les faits divers, propices à l'émergence d'expressions inscrites dans le contexte spatio-temporel de l'événement médiatisé. Ancré dans la culture francophone (Lits, 2001), le fait divers se caractérise par une diversité structurelle et thématique forte, qui rend difficile sa classification parmi les genres journalistiques classiques. L'ancrage majeur du fait divers dans l'univers médiatique matérialise la condition même de son existence et de sa diffusion (Dubied, 2001). Malgré cette forte diversité, la linguiste Gonon (2012) observe que les reprises narratives, lexicales et syntaxiques entre les récits révèlent un figement $d u$ fait divers. Ces reprises donnent lieu à une réappropriation des discours circulant et favorisent ainsi le recours à l'allusion (Authier-Revuz, 2020). La porosité entre le fait divers et les productions discursives qui lui sont antérieures situe au cœur de l'étude de ce genre la notion d'interdiscours (Pêcheux, 1969). Lorsqu'un terme déjà associé à un moment marquant est employé pour désigner un nouvel événement ou un acteur, les représentations attachées à ce moment marquant tendent à être réactualisées. C'est ce que Moirand nomme «la mémoire des 
mots » (Moirand, 2007b). Structuré par un discours narratif, le fait divers est centré autour d'un acteur sujet (Adam, 1996) responsable de l'action relayée. Lorsque cette action est un crime, celui qui en est l'auteur est désigné par des noms axiologiques négatifs tels que ogre, tueur, monstre, loup-garou, etc. Le caractère générique de la dénomination est alors atténué par la restriction de catégorie qu'opère la mention fréquente du lieu de l'événement en complément du nom. Le syntagme se construit alors selon la forme [article défini + nom commun $+d e+$ toponyme]. L'article défini présuppose l'existence d'un référent unique - appartenant à une zone géographique spécifique. La permanence de la structure du syntagme nominal établit néanmoins entre les faits de même type une "résonance événementielle » (Kalinic, 2005). À l'instar des mots événements dans la presse, la nomination des acteurs des faits divers provoque des effets de mémoire dépassant le sens de l'expression et relevant de la mémoire interdiscursive médiatique (Moirand, 2007b). Ces processus de nomination renforcent les liens entre des événements relatés n'impliquant pas nécessairement les mêmes protagonistes, alors que la dénomination se fonde sur une unicité référentielle. L'analogie entre deux événements peut conduire au réinvestissement d'une expression datée pour un événement contemporain. Par exemple, en 1978, le tueur en série belge René Hebrant avait été médiatisé et surnommé «le monstre des Ardennes ». Au début des années 2000, les meurtres de Michel Fourniret conduisent à nouveau à la médiatisation d'un tueur en série par la désignation le monstre des Ardennes. L'analogie entre les deux meurtriers que permet l'usage du syntagme peut conférer au fait divers actuel les attributs du précédent et indiquer parallèlement le genre journalistique privilégié. Dans le présent article nous cherchons à analyser les mécanismes d'actualisation d'une expression datée dans le discours journalistique, à travers l'exemple du réinvestissement du syntagme la Bête du Gévaudan.

2 En France, l'expression Bête du Gévaudan a émergé en 1764 et a largement été relayée par la presse écrite pour désigner un animal qui a tué, entre 1764 et 1767, des dizaines de personnes sur le territoire français (Moriceau, 2008). À la fin du xxe siècle, le retour du loup, espèce protégée, sur le territoire français, s'accompagne de la médiatisation d'attaques de troupeaux ovins dans deux régions : les Vosges et le Haut-Var. Dans les deux cas, le prédateur recherché est désigné par le syntagme la bête de + toponyme [Vosges/Haut-Var], entrant ainsi en résonnance avec la référence aux auteurs de crimes d'une part et à l'expression datée Bête du Gévaudan d'autre part. À travers une étude comparative d'un corpus de 23 articles du quotidien régional Nice-Matin et du quotidien national Le Monde, respectivement diffusés dans la zone où le loup est revenu en 1992 et sur l'ensemble du territoire, nous avons observé les variations de traitement de ces événements et les modalités de réinvestissement de l'expression la Bête du Gévaudan. Dans quelle mesure l'analogie entre les expressions la bête des Vosges/du HautVar et la Bête du Gévaudan constitue-t-elle, dans la presse contemporaine, une référence aux récits de faits divers relatifs à la Bête du Gévaudan?

3 Nous décrirons tout d'abord les spécificités du fait divers de la Bête du Gévaudan, pour ensuite analyser l'émergence des expressions la bête des Vosges dans Le Monde et la bête du Haut-Var dans Nice-Matin. 


\section{Le fait divers de la Bête du Gévaudan raconté par la presse}

4 Les attaques attribuées à la Bête du Gévaudan ont été relayées par plusieurs journaux français entre 1764 et 1767. Le Courrier d'Avignon et La Gazette de France ont respectivement publié 66 et 14 articles à ce sujet (Mazel \& Garcin, 2008). L'inscription dans la durée de l'affaire et les rebondissements qui l'ont structurée ont contribué à créer une tension dans la construction même du récit, à travers un des aspects fondamentaux du fait divers : la relation de causalité (Barthes, 1964). Barthes distingue le roman policier, dans lequel l'énigme s'étire dans le temps, du fait divers qui se caractérise par son immanence et l'identification rapide, troublante, de la cause de l'événement narré. Dans le cas des attaques du Gévaudan, l'absence d'identification certaine du prédateur prive l'événement raconté de sa cause. La répétition d'attaques de même type a néanmoins renforcé l'immanence du fait divers malgré son inscription dans la durée. Les victimes (enfants et jeunes filles) et les conditions macabres de leur mort ont contribué à maintenir une tension quant à la recherche du prédateur et à la relation de causalité qui structurait le récit. L'identification répétée du prédateur supposé être à l'origine des attaques a alimenté l'imaginaire autour de l'identité de l'acteur sujet (Moriceau, 2008) et a ancré dans la mémoire collective les propriétés attribuées à la Bête du Gévaudan. En raison de l'ancrage historique du récit des attaques (Moriceau, 2008), l'expression Bête du Gévaudan réfère aujourd'hui de façon systématique au prédateur présent sur le territoire entre 1764 et 1767 . Remplacer le nom bête par animal, par exemple, affaiblit la référence. L'expression la Bête du Gévaudan constitue un segment stabilisé et a de ce fait acquis un degré fort de figement au cours du temps. L'usage de la forme [la Bête de + toponyme] pour référer à un prédateur ayant attaqué des humains ou des animaux est susceptible de fournir d'une part au lecteur un indice quant au genre journalistique privilégié - celui du fait divers - et d'autre part de conférer à l'acteur sujet du récit des propriétés analogues à celles de la Bête du Gévaudan. Peu après le fait divers du Gévaudan, des attaques qui ont lieu dans les Cévennes et à Orléans conduisent à réinvestir l'expression en modifiant le toponyme. Dans le Journal du Gard, l'extrait d'un article publié le 21 octobre 1809 illustre le phénomène de réinvestissement de l'expression: «Depuis quelques jours, un animal féroce a répandu la terreur dans le premier arrondissement du Gard. Comme autrefois la Bête du Gévaudan, la Bête des Cévennes exerce aujourd'hui ses ravages dans cette contrée ». L'analogie explicite entre les deux expressions confère ici au nouvel acteur sujet - la Bête des Cévennes - les propriétés de la Bête du Gévaudan. La notoriété du récit du Gévaudan est encore soulignée plusieurs dizaines d'années après les événements. Par exemple, dans le quotidien régional Le Moniteur, un journaliste consacre en 1866 un article aux moyens envisagés pour traquer l'animal et souligne l'influence de ce fait divers sur la littérature et l'imaginaire collectif:

(1) À la fin du xVIII', la Bête du Gévaudan fit beaucoup parler d'elle; elle obtint "presque autant de renommée qu'un conquérant ». Elle a été célébrée en vers et en prose, mise en chansons et naturellement on en vint à exagérer ses méfaits. De nos jours, elle a été mise en roman par Elie Berthet.

Le Moniteur, « La Bête du Gévaudan », le 17 octobre 1866

5 Au cours des deux derniers siècles, les événements qui se sont produits dans le Gévaudan ont fait l'objet de travaux historiques (Ménatory, 1976; Pourcher, 2006 ; Moriceau, 2008) et d'écrits de fiction (Berthet, 2019; Roche, 2014). Cela a contribué à 
ancrer dans la mémoire interdiscursive (Moirand, 2007a) le récit et l'expression, de sorte que l'explicitation de la référence ne soit plus nécessaire pour établir un lien entre le syntagme la bête $d u$ + toponyme et la Bête du Gévaudan. Dans le contexte contemporain, la médiatisation du retour du loup en France en 1992 a été propice au réinvestissement d'événements et de mythes datant des périodes où le loup était présent sur le territoire. C'est notamment le cas pour deux événements de prédation qui s'inscrivent dans la durée dans les Vosges (1994-1995) et le Haut-Var (1993-2001).

\section{La bête des Vosges dans Le Monde, en 1994 et 1995}

6 Parallèlement au retour du loup dans le sud-est de la France, des actes de prédation ont lieu dans les Vosges en 1994. L'animal qui en est à l'origine est rapidement identifié, ce qui rend possible le passage d'un récit d'abord marqué par l'incertitude de la cause à un récit fondé sur la traque du prédateur. Le département des Vosges avait déjà connu, en 1977, des actes de prédation de la part d'un animal qui n'a jamais été identifié. L'incertitude de l'identité du prédateur a conduit, à cette époque, au développement d'une multitude d'hypothèses. Ces événements avaient bénéficié d'une couverture médiatique régionale et nationale et l'animal avait alors déjà été désigné par l'expression bête des Vosges, comme en témoigne l'extrait d'un article du quotidien SudOuest, publié le 18 juin 1977 dans la rubrique Faits Divers : «La disparition subite de la "bête des Vosges", qui a sévi pendant plus d'un mois dans les Vosges lorraines, faisant 150 victimes, pour la plupart de paisibles moutons, n'a pas empêché l'enquête de se poursuivre et les chasseurs viennent de déposer plainte contre X à Epinal $»^{1}$. En explicitant l'appartenance à un discours autre et une mise à distance journalistique, la modalisation autonymique (Authier-Revuz, 2020) souligne le caractère circulant de l'expression bête des Vosges. La difficulté à identifier l'auteur des actes de prédation contribue à établir une résonnance événementielle entre le fait divers actuel et celui de la Bête du Gévaudan.

7 Les actes de prédation qui se déroulent dans les Vosges en 1994 et 1995 bénéficient d'une couverture médiatique importante dans Le Monde, qui publie 9 articles à ce sujet dans les rubriques "Chroniques » (3 articles), "Société », dans des articles d'analyse (3 articles) et de faits divers ( 2 articles). La nature des sections privilégiées témoigne d'une volonté de situer le feuilleton au cœur de l'actualité. Si le récit des attaques qui ont eu lieu en 1977 avait déjà conduit à désigner le prédateur par l'expression la bête des Vosges, la multiplication d'incidents dans le contexte de recolonisation du territoire par le loup peut favoriser le réinvestissement d'expressions datées telles que la Bête du Gévaudan mais aussi, plus largement, d'un ensemble de récits renvoyant aux siècles où le loup était présent en France.

\subsection{Le rôle de la mémoire discursive dans la construction du récit}

Le Monde façonne le récit des attaques qui ont lieu dans les Vosges en recourant de façon répétée au savoir encyclopédique de son lectorat. De l'évocation de comptines pour enfants à la citation de la fable de La Fontaine "Le Loup et le Chien", les journalistes structurent le récit à travers le réinvestissement d'événements, d'œuvres et de documents historiques qui entrent en interaction avec les événements actuellement relayés. C'est ainsi qu'est diffusée l'information de la présence du loup 
dans les Vosges dans un article publié le 21 novembre 1994: «Tel le furet de la chanson, le loup reparaît en mai 1994 en un lieu où on ne l'attendait pas : les Vosges »².

Les références littéraires façonnent dans la durée un véritable feuilleton à partir des événements relatés dans les tribunes publiées en 1994 et 1995. La nomination du loup par le prénom Arsène est un moyen pour les journalistes de construire une image positive du loup en usant de l'imaginaire des ouvrages de Maurice Leblanc, qui met en scène Arsène Lupin, un gentleman-cambrioleur : «Ainsi donc Arsène, notre bon vieil Arsène qui croqua d'une dent allègre quatre-vingt-deux brebis et dupa pendant des mois la fine fleur de la louveterie française, est-il probablement mort ${ }^{3}$. La référence reste alors implicite et instaure avec le lectorat un univers de connaissance partagée.

D'un point de vue historique, la presse fait d'emblée explicitement référence aux attaques qui avaient eu lieu à la fin des années 1970 : « Les petits enfants en frissonnent délicieusement de peur au fond de leurs lits et, dans les cafés, les chasseurs et les agriculteurs ne parlent plus que d'elle. La bête des Vosges qui avait semé la terreur dans la région il y a vingt ans serait revenue $»^{4}$. La référence au fait divers datant de 1977 est explicite, tandis que l'analogie qui peut être perçue entre les expressions bête du Gévaudan et bête des Vosges n'est pas soulignée. Néanmoins, cet extrait se trouve dans le premier article publié sur les actes de prédation des Vosges et construit d'emblée un monde représenté proche de celui de contes mettant en scène le loup. L'énoncé frissonner délicieusement de peur au fond de leurs lits ancre le fait divers dans un univers de fiction, renforcé par l'assimilation de l'animal actuellement présent dans les Vosges à celui qui avait été recherché en 1977. Dans un article publié le $1^{\text {er }}$ novembre 1995, le journaliste établit à nouveau une comparaison entre les deux faits divers ayant marqué les Vosges et utilise l'expression bête des Vosges :

(2) Très vite, donc, le loup se fait connaître par sa faim. Et rappelle la sauvagerie que causa, à la fin des années 70, la désastreuse et mystérieuse "bête des Vosges" (jamais retrouvée). [...] Fin décembre, il compte à son bilan plus de quatre-vingts victimes : brebis, béliers, génisses, poulain et pouliche.

Le Monde, « Le loup des Vosges, l'État et les éleveurs », 11 janvier 1995

11 Le vocabulaire axiologique négatif sauvagerie, désastreuse, victime est propice au renvoi, par la mémoire sémantique (Moirand, 2007b), à des représentations associées au loup lorsque le statut de l'animal était celui de nuisible.

Le réinvestissement de références littéraires et historiques pour façonner le récit des Vosges relève jusqu'ici d'indices localisés, dont le degré d'explicitation par les journalistes est variable mais aisément perceptible pour le lecteur. Dans les récits de prédation des Vosges, les termes employés en cotexte de l'expression bête des Vosges peuvent contribuer, par la mémoire dont ils sont porteurs, à rapprocher le récit de Vosges de celui du Gévaudan.

\subsection{Le rôle du cotexte dans le processus d'actualisation d'une expression datée}

L'analogie de l'expression [la bête de + nom de lieu] avec le syntagme la Bête du Gévaudan est susceptible d'être renforcée par l'environnement textuel. Nous nous intéressons à l'impact que peut avoir le cotexte dans la construction de la représentation du référent et dans l'actualisation d'un syntagme daté. Le prédateur est désigné par les expressions bête des Vosges et bête de Senonges à 13 reprises. Le cotexte est marqué par l'isotopie de la 
violence d'une part, avec les termes terreur, massacre, désastreuse, carnage et par l'isotopie de l'incertitude d'autre part avec mystérieuse et mystère:

(3) Le mois suivant, la bête des Vosges se manifeste par un massacre chez un éleveur de moutons de Dommartin-lès-Vallois (trois brebis égorgées et cinq autres blessées, qu'il faut achever). L'Office national de la chasse (ONC) mobilise ses gardes et le lieutenant de louveterie local pour tâcher de neutraliser le fauve. En vain. Une pouliche et quatre brebis font encore les frais de l'errance sauvage du loup, qui se montre particulièrement rusé.

Le Monde, « Le loup (ou la louve) des Vosges », 21 novembre 1994 encore par le substantif victime attribuent des propriétés anthropomorphiques aux animaux tués par le loup. Dans l'extrait de l'article publié le 11 janvier 1995, l'usage de l'expression ôter la vie dans l'énoncé on n'a plus de nouvelles [du loup] depuis qu'il ôta la vie, le 5 décembre à Esley (commune voisine), à " Guimauve », une jeune pouliche appartenant à M. Lassausse opère une analogie avec des faits divers mettant en scène des loups anthropophages et dont la Bête du Gévaudan est le symbole. L'extrait d'un article de la Gazette de France, publié en 1764 et relatant les attaques de la Bête du Gévaudan sur des humains en témoigne :

(4) Il paraît depuis deux mois dans cette Province, aux environs de Langogne \& de la Forêt de Mercoire, une bête farouche qui répand la consternation dans toutes les campagnes. Elle a déjà dévoré une vingtaine de personnes, surtout des enfants et particulièrement de jeunes filles ; il n'y a guère de jours qui ne soient marqués par quelque nouveau désastre.

La Gazette de France, "Extrait d'une lettre écrite par Marvejols dans le Gévaudan ", 23 novembre 1764 de l'action au milieu rural. Dans l'énoncé Le "Bois de curé", deux petits kilomètres carrés de forêt giboyeuse, est le dernier repaire connu du loup de Senonges, les indices spatiaux indiqués par les syntagmes forêt giboyeuse et Bois de Curé confèrent à la narration un caractère daté et renforcent ainsi l'analogie avec le récit du Gévaudan.

L'usage conjoint d'un vocabulaire daté et d'un vocabulaire axiologique construit un fait divers imprégné du récit de la Bête du Gévaudan. La mise en récit de la traque du loup se fonde sur le réinvestissement d'un univers mythique non simplement lié à la Bête du Gévaudan mais renvoyant plus généralement aux récits d'interactions entre les hommes et les loups au cours des siècles précédents. Le Monde multiplie les références pour façonner le feuilleton consacré au loup présent dans les Vosges et mêle au récit de fait divers des récits de fiction. La traque du loup est ainsi teintée d'ironie. Le caractère implicite de la référence établit une connivence avec le lectorat, auquel on suppose de fait une culture littéraire et historique.

Dans Nice-Matin, la couverture médiatique des actes de prédation qui ont lieu dans le Haut-Var s'inscrit plus encore dans la durée. Cette inscription dans la durée, associée à l'incertitude de l'identification du prédateur, est susceptible de rapprocher davantage le récit médiatique régional de celui, historique, de la Bête du Gévaudan. 


\section{La bête du Haut-Var dans Nice-Matin} se structure autour de la recherche de l'identité du prédateur, qui ne sera connue qu'en 2001. Le feuilleton est de ce fait marqué par l'incertitude, rendant ainsi possibles les analogies avec des événements antérieurs et la multiplication des opérations de catégorisation pour désigner l'acteur sujet du récit.

\subsection{Le rôle de la mémoire discursive dans la construction du récit}

À la différence du Monde, le récit des actes de prédation dans Nice-Matin est peu construit par le réinvestissement de références littéraires et historiques. C'est à travers des expressions telles que loup y es-tu ${ }^{5}$; le loup dans la bergerie $e^{6}$ ou encore dans la gueule $d u$ loup $^{7}$ que la référence aux récits de fiction s'opère. Ces expressions, intégrées aux titres des articles, attestent de l'adoption d'un ton léger pour relayer les actes de prédation, cependant contrebalancé par la juxtaposition d'un vocabulaire relevant de l'isotopie de la violence comme, par exemple, dans le titre de l'article publié le 16 octobre 1996: "Le loup dans la bergerie. Encore neuf moutons égorgés dans le HautVar ». L'adverbe encore souligne l'aspect répétitif des prédations et renforce, parallèlement à la quantification du nombre de proies, le caractère violent des modalités de l'attaque (égorgement). Entre 1993 et 2001, 14 articles sont publiés au sujet des événements qui ont lieu dans le Haut-Var. L'usage répété de l'expression la bête du Haut-Var (15 occurrences), en répondant à la structure syntaxique privilégiée dans les faits divers pour désigner l'auteur d'un crime, contribue à typifier le genre du fait divers, alors que la nature du cotexte et les multiples catégorisations du prédateur fondent l'unicité du récit.

\subsection{Le rôle de la catégorisation dans le processus d'actualisation d'une expression datée}

21 L'absence d'identification certaine du prédateur lors de la médiatisation des incidents répétés est propice à la diversification des mots utilisés pour nommer l'animal. Parallèlement à la désignation du prédateur par le syntagme bête du Haut-Var, les journalistes réfèrent à l'animal par les termes (mystérieux) égorgeur de mouton/d'ovin (6 occurrences) ; féroce canidé (2 occurrences), ou encore dents de la montagne, explicitant ainsi conjointement l'incertitude de l'identité du prédateur et l'insécurité que sa présence non maîtrisée génère. Le vocabulaire privilégié fait écho à celui qui est employé lors de l'enquête et de la traque de la Bête du Gévaudan, comme le montre cet extrait issu d'une lettre d'un représentant local de l'autorité royale en 1764 et cité par l'historien Moriceau (2008) : «Depuis plus de six mois [...] une bête féroce, qui rôde dans nos montagnes du Vivarais, voisines de celles du Gévaudan, a fait éprouver aux habitants de ce canton l'effet de sa voracité ». Dans Nice-Matin, l'usage de l'adjectif féroce au sein d'un paragraphe qui souligne l'incertitude de l'identification du prédateur attribue à la bête du Haut-Var des propriétés analogues à la Bête du Gévaudan : 
(5) Loup semi-domestique, lynx, chien errant ou sauvage, l'animal continue donc à alimenter tous les fantasmes et toutes les suppositions. Il n'en demeure pas moins qu'à l'approche de la saison estivale, les éleveurs de Trigance ont peur. Avec ce féroce prédateur en liberté, l'été promet en effet d'être particulièrement sanglant pour les troupeaux du plateau de Canjuers.

Nice-Matin, « Dans la gueule du loup », 21 mars 1995

Si le journaliste marque une distance avec les hypothèses portant sur l'identité du prédateur en les envisageant en tant que croyances, les occurrences des termes peur, féroce et sanglant situent le discours du côté du pathos. Les catégorisations axiologiques du prédateur construisent son image selon des propriétés proches de celles attribuées à la Bête du Gévaudan et façonnent plus largement l'appartenance du récit au genre du fait divers.

\subsection{Le rôle du cotexte dans le processus d'actualisation d'une expression datée}

L'analogie entre les opérations de catégorisation du prédateur dans le récit du Gévaudan et du Haut-Var est renforcée par l'appartenance de ces deux récits à un même genre de discours. Dans Nice-Matin, on retrouve une isotopie de l'insécurité propre à la mise en scène du récit selon les codes textuels, syntaxiques et lexicaux du fait divers. Dans le Haut-Var, les attaques font l'objet d'analyses scientifiques que les journalistes relaient selon les standards de l'enquête policière : «Il arrive parfois que les enquêteurs aient besoin du coup de pouce de l'informatique pour remonter une piste et arriver au coupable. Ainsi, la brigade de recherche de Draguignan, placée sous les ordres de l'adjudant-chef Bertrand, va utiliser des banques de données informatiques pour traquer la bête du haut Var $»^{8}$. La catégorisation du loup par le substantif coupable, dans un contexte où le vocabulaire utilisé se rapporte au domaine judiciaire, renvoie au fait divers criminel. L'axiologie négative, qui participe à créer un climat d'incertitude et d'insécurité dépasse ici la caractérisation unique de l'acteur sujet. Il est question de "carnages ovins ${ }^{9}$, "secteur maudit», "mauvais cauchemar $»^{10}$, «nouvelle tuerie», «été sanglant $»^{11}$, «massacres $»^{12}$. Ces catégorisations générales rapprochent la description de la scène de prédation de celle d'une scène de crime. L'analogie est appuyée par la mention, dans la description, d'espaces urbanisés : «La Bête du Haut-Var aurait été identifiée par deux promeneurs après une nouvelle tuerie dans le quartier de Saint-Mayme, sur la commune de Trigance ». La catégorisation anthropomorphique des proies participe de cette mise en scène lors de descriptions à la fois quantitatives et qualitatives des actes de prédation :

(6) Au cours de l'avant-dernière nuit, la bête a encore fait son œuvre, dévorant une brebis, en tuant cinq et blessant grièvement trois autres moutons. Ce bilan n'est pas complet, les éleveurs cherchent encore d'autres victimes. Ce nouveau carnage intervient au lendemain d'une réunion d'information provoquée par la fédération des chasseurs du Var en mairie de Comps.

Nice-Matin, « Le loup dans la bergerie », 16 octobre 1996

L'emploi des mots victime et grièvement, couramment utilisés pour référer à des humains attribue à la proie un caractère anthropomorphique. Dans ce contexte, la structure même [article défini + nom commun + de + toponyme] typifie le genre de discours du fait divers, alors que la modification du toponyme inscrit le syntagme dans l'actualité. Le recours aux codes du fait divers conduit à rapprocher la scène de 
prédation d'une scène de crime et favorise le réinvestissement des propriétés d'incertitude et d'insécurité des événements passés dans le contexte contemporain.

\section{Conclusion}

La médiatisation des actes de prédation dans les Vosges et le Haut-Var s'accompagne du réinvestissement du récit de la Bête du Gévaudan, notamment en raison du caractère incertain de la traque (pour les Vosges) et de l'identité (pour le Haut-Var) du prédateur. La désignation de l'animal par les expressions la bête des Vosges ou la bête du Haut-Var établit une relation d'unicité entre le référent et sa dénomination. Nous avons observé une tension entre le maintien du caractère daté du syntagme, porteur de la mémoire discursive, et la revitalisation par la modification du toponyme. La relation de dénomination suppose une préexistence $d u$ référent à la formation du récit. L'actualisation de l'expression attribue au référent des propriétés façonnées par les usages antérieurs de la structure la bête de + toponyme, et notamment par la dénomination la Bête du Gévaudan.

Dans Le Monde comme Nice-Matin, l'interdiscours est un moyen pour le journal d'assurer la visée de captation (Charaudeau, 2005), que ce soit en développant un univers de connaissance partagée ou en sollicitant l'affect du lectorat. Dans Le Monde, le récit des Vosges se fonde sur une ambiguïté entre le monde représenté que les journalistes façonnent à partir de faits avérés, et les mondes mythiques auxquels les textes se réfèrent pour alimenter la mise en scène de la prédation. Nice-Matin confère au récit le statut de fait divers en empruntant les codes qui caractérisent ce genre de discours. Par l'emploi répété de l'expression bête du Haut-Var, il renforce l'attribution au loup de propriétés usuellement associées aux criminels dans les faits divers.

Dans le processus d'actualisation d'une expression datée, il se créée une tension dans le discours journalistique entre deux enjeux : conserver le caractère daté de l'expression réinvestie (ici la Bête du Gévaudan) pour l'univers discursif qu'elle fait émerger et assurer le renouvellement sémantique du syntagme modifié (ici la bête des Vosges et la bête du Haut-Var) pour situer cet univers dans le présent. De cette façon, l'univers ancien ainsi propulsé dans l'actualité confère dès son premier usage une dimension vieillie à l'expression modifiée.

\section{BIBLIOGRAPHIE}

ADAM, J.-M., 1996, Le récit, Paris, Presses Universitaires de France.

AUTHIER-REVUZ, J., 2020, La Représentation du Discours Autre : principes pour une description, Berlin, De Gruyter.

BARTHES, R., 1964, « Structure du fait divers », Essais critiques, Paris, Éditions du Seuil.

BERTHET, E., 2019, [1858], La Bête du Gévaudan, Paris, Libretto. 
CHARAUDEAU, P., 2005, Les médias et l'information. L'impossible transparence du discours, Louvain-laNeuve, De Boeck-Ina.

DUBIED, A., 2001, « Invasion péritextuelle et contaminations médiatiques. Le "fait divers", une catégorie complexe ancrée dans le champ journalistique ", Semen, 13, Genres de la presse écrite et analyse de discours, https://journals.openedition.org/semen doi : https://doi.org/10.4000/semen. 2633

GONON, L., 2012, Le fait divers criminel dans la presse quotidienne française du XIX siècle, Paris, Presses Sorbonne Nouvelle.

KALINIC, A., 2005, « Faits divers dans les journaux télévisés : récits mythiques ? », Les Cahiers du journalisme, 14, Lille, Centre de Recherche de L'École Supérieure de Journalisme de Lille, p. 174-187.

LITS, M., 2001, « Le fait divers : un genre strictement francophone? ", Semen, 13, Genres de la presse écrite et analyse de discours, https://journals.openedition.org/semen doi : https://doi.org/ $10.4000 /$ semen.2628

MAZEL É., GARCIN, P.-Y., 2008, La Bête du Gévaudan. À travers 250 ans d'images, Marseille, Éditions Gaussen.

MOIRAND, S., 2007a, Les discours de la presse quotidienne : observer, analyser, comprendre, Paris, Presses Universitaires de France.

MOIRAND, S., 2007b, « Discours, mémoires et contextes : à propos du fonctionnement de l'allusion dans la presse », Corela, HS-6, Cognition, discours, contextes, <https:// journals.openedition.org/corela>, doi:10.4000/corela.1567

MENATORY, G., 1976, La Bête du Gévaudan. Histoire, légende, réalité, Paris, FeniXX réédition numérique.

MORICEAU, J.-M., 2008, La Bête du Gévaudan : 1764-1767, Paris, Larousse.

PÊCHEUX, M., 1969, Analyse automatique du discours, Paris, Dunod.

POURCHER, P., 2006 [1889], La Bête du Gévaudan. Véritable fléau de Dieu, Marseille, Éditions Jeanne Laffitte.

ROCHE, G., 2014, Gévaudan. Le Roman de la Bête, Clermont-Ferrand, Editions de Borée.

\section{NOTES}

1. Sud-Ouest, «La "bête des Vosges" : un loup du Nevada dressé ? ", 18 juin 1977.

2. Le Monde, « Le loup (ou la louve) des Vosges», 21 novembre 1994.

3. Le Monde, « La mort du loup ", $1^{\text {er }}$ février 1995.

4. Le Monde, «Un loup recherché dans les forêts de l'Est ", $1^{\mathrm{er}}$ août 1994.

5. Nice-Matin, « Haut-Var : loup y es-tu? », 26 janvier 2001.

6. Nice-Matin, «Le loup dans la bergerie. Encore neuf moutons égorgés dans le Haut-Var », 16 octobre 1996.

7. Nice-Matin, «Dans la gueule du loup. La Bête du Haut-Var aurait été identifiée par deux promeneurs après une nouvelle tuerie », 21 mars 1995.

8. Nice-Matin, «L'empreinte de la bête », 2 novembre 1994.

9. Nice-Matin, « Dans la gueule du loup », 21 mars 1995.

10. Nice-Matin, « Un loup dans l'Artuby : un lynx repéré à la Verdière », 20 août 1993. 
11. Nice-Matin, « Dans la gueule du loup », 21 mars 1995.

12. Nice-Matin, «Un loup dans l'Artuby : un lynx repéré à la Verdière », 20 août 1993 ; «Le loup dans la bergerie », 16 octobre 1996.

\section{RÉSUMÉS}

Le discours de presse est propice à l'émergence et à l'usage répété d'expressions pour référer à un événement spécifique. La diminution de la couverture médiatique accordée à l'événement peut conduire à une diminution, voire à une disparition de l'expression et conférer ainsi à cette dernière un caractère daté. Du fait de leur fort ancrage spatial et temporel, les faits divers se présentent comme un terrain favorable pour l'étude des phénomènes d'émergence et de disparition des usages. L'emploi répété de la structure [article défini + nom commun + de + toponyme] pour désigner l'auteur d'un crime est typique du genre du fait divers (le monstre des Ardennes, le loup garou de Dôle, etc.). À travers l'exemple de l'expression la Bête du Gévaudan, qui a désigné entre 1764 et 1767 un prédateur ayant attaqué plusieurs dizaines de personnes, nous nous sommes intéressée aux mécanismes de réinvestissement d'une expression datée dans le discours contemporain. Nous avons constitué un corpus de 23 articles issus du Monde et de NiceMatin, relayant les actes de prédation des loups au début des années 1990, pour identifier les liens qu'entretenaient les expressions la bête des Vosges et la bête du Haut-Var avec l'expression la Bête du Gévaudan. Nous avons montré que dans le processus d'actualisation des syntagmes la bête des Vosges et la bête du Haut-Var, le caractère daté de l'expression la Bête du Gévaudan est réinvesti pour façonner un univers mythique dans lequel prennent place les actions du récit contemporain. De ce fait, l'expression modifiée porte d'emblée une dimension historique.

Media discourses favour the emergence and the frequent use of events related expressions. The decrease of the media coverage of a specific event can lead to a diminution or a disappearance of the expression. Crime news are particularly associated with the emergence of such expressions, due to a strong spatial and temporal contextualisation. Syntactic structure [the + noun + of + toponym] is used to name a criminal in crime news: the monster of Ardennes; the werewolf of Dôle, etc. (le monstre des Ardennes, le loup garou de Dôle, etc.). Focusing on a specific expression (The beast of Gevaudan; la bête du Gévaudan) related to media coverage of predator's attacks that caused the death of dozens of people in France between 1764 and 1767, we analysed how an old expression can be reused in contemporary discourses. We collected 23 articles from two French newspapers (Le Monde and Nice-Matin) and analysed how the expressions the beast of Vosges (la bête des Vosges) and The beast of Haut-Var (la bête du Haut-Var) were related to the expression the Beast of Gevaudan (la Bête du Gévaudan). We showed that contemporary expressions maintained spatial and temporal properties of the former crime news to shape a mythical universe in which current actions take place.

\section{INDEX}

Mots-clés : catégorisation, interdiscours, presse écrite, fait divers

Keywords : categorisation, interdiscourse, printed press, crime news 
AUTEUR

\section{MARIE CHANDELIER}

Université Côte d'Azur, Laboratoire Bases, Corpus, Langage (UMR 7320) 